\title{
Destinazioni minori \\ e sviluppo turistico sostenibile. II progetto EDEN nella Regione Lombardia
}

VIVIANA CALZATI

\begin{abstract}
Obiettivo del paper: Il presente lavoro si propone di analizzare gli elementi che caratterizzano alcuni territori, non coinvolti dal turismo di massa, come possibili destinazioni minori di eccellenza in una visione di sviluppo locale sostenibile. L'ipotesi del lavoro è che la sostenibilità del modello di sviluppo è essa stessa un valore capace di agire sulla competitività turistica delle destinazioni minori che non solo non possono essere identificate come territori marginali, ma posizionarsi al contrario come distinct areas.

Metodologia: La metodologia utilizzata consiste in un'analisi della letteratura sul tema e nella raccolta di dati ricavati da fonti pubbliche e interviste dirette ai responsabili del progetto EDEN nelle destinazioni lombarde.

Risultati: L'analisi condotta evidenzia come il progetto comunitario sia riconosciuto strumento capace di aumentare la visibilità di una destinazione minore, ma non ancora come opportunità per attivare la condivisione e lo scambio di buone pratiche in tutto il territorio $U E$.

Limiti della ricerca: Il numero ridotto dei casi analizzati e l'assenza di un set di indicatori in grado di misurare il reale impatto sulla comunicazione turistica rappresentano un limite della ricerca.

Implicazioni pratiche: Il presente lavoro lascia trasparire potenziali implicazioni pratiche per la valutazione della coerenza tra gli obiettivi del progetto EDEN e i suoi risultati e per una governance del territorio in un'ottica di valorizzazione e partecipazione.

Originalità del lavoro: L'aspetto maggiormente originale consiste nell' aver individuato, nelle destinazioni minori, aree capaci di promuovere modelli di sviluppo turistico sostenibile considerati eccellenti, in cui la qualità territoriale e la marginalità assumono connotazione positiva sino a diventare fattori distintivi.
\end{abstract}

Parole chiave: sviluppo sostenibile, destinazioni minori, qualità, progetto EDEN

Si ringraziano per la collaborazione e la cortese disponibilità il Comune di Monte Isola, il Parco Regionale del Mincio e il Dipartimento per lo sviluppo e la competitività del turismo in particolare il Dott. Lucio D'Amore Capo Progetto EDEN Relazioni comunitarie e internazionali

** Assegnista di ricerca di Economia e Gestione delle Imprese - Università degli Studi di Perugia

e-mail: viviana.calzati@unipv.it 
Purpose of the paper:This paper aims to study the features of slow territories as possible minor benchmark destinations in a new vision of local sustainable development. The hypothesis behind the work states that the sustainability of the development model is in itself a value capable of influencing the tourism competitiveness of minor destinations, which cannot be identified as marginal territories, but which can, on the contrary, be viewed as distinct areas.

Methodology: The paper analyses literature on the subject and collects data from public sources and interviews held by those in charge of the EDEN project in Lombardy.

Findings: The analysis highlights the way in which the Community project has been recognised as being an instrument, capable of increasing the visibility of a minor destination, but not so far as being an opportunity, which will encourage good practice to be shared and exchanged throughout EU territory.

Research limits: Research was limited by the restricted number of cases analysed and the lack of a set of indicators capable of measuring the impact on tourist communication.

Practical implications: This work reveals potential, practical implications not only for the assessment of consistency between the EDEN project objectives and its results, but also for the management of the territory from the point of view of enhancement and participation.

Originality of the study: The most original aspect of the work consists of the identification of minor destinations as areas capable of promoting excellent models of sustainable tourist development, in which their slow emergence and marginal condition can be regarded positively, so as to become their distinctive factor.

Key words: sustainable development, minor destinations, quality, EDEN project

\section{Introduzione}

Il presente lavoro partendo da una nuova visione di territorio, che attribuisce valore alle identità, al capitale sociale e al patrimonio culturale autoctono, analizza la relazione tra sviluppo turistico sostenibile e destinazioni minori.

In questo contesto il paper mira inizialmente a caratterizzare i principali tratti di un percorso di sviluppo sostenibile attraverso l'analisi di territori minori. In tali territori si individuano nuove traiettorie di sviluppo in cui l'identità, la storia locale, il capitale sociale, il patrimonio culturale, la qualità e la bellezza possono divenire innovativi fattori strategici di sviluppo turistico sostenibile.

Successivamente il paper analizzerà la Comunicazione della Commissione Europea 'L'Europa, la destinazione turistica $n .1$ del mondo. Un nuovo quadro di riferimento politico per il turismo in Europa (2010) che ha evidenziato la necessità di creare un marchio europeo "Turismo di qualità" finalizzato, da un lato a stimolare le destinazioni ad applicare pratiche sostenibili e dall'altro a rafforzare l'immagine dell'Europa come destinazione turistica di qualità.

Ulteriore approfondimento riguarda le origini e gli obiettivi del Progetto Destinazioni Europee di Eccellenza (o EDEN), acronimo di European Destinations of ExcelleNce), iniziativa promossa dalla Commissione Europea al fine di promuovere in tutta l'Unione Europea modelli di sviluppo turistico sostenibile. Il focus sarà sulla Regione Lombardia in cui due territori si sono classificati come 
destinazioni EDEN dal 2007 al 2011: il comune di Monte Isola (BS) e la Riserva Naturale Valli del Mincio costituisce l'ultima parte del lavoro.

\section{Le destinazioni minori tra qualità e sostenibilità}

I territori minori ritenuti ai margini dei sistemi forti e consolidati individuano nel territorio un fattore competitivo importante in cui si rafforzano interessi e identità collettivi e in cui la sua coesione sociale rappresenta valore aggiunto capace di promuovere distretti di qualità. Oggi viviamo una fase di ri-personalizzazione dell'economia e quindi anche dello spazio fisico, che pone al centro i significati elaborati dalle persone e conferiti ai luoghi (Bonomi e Rullani, 2005).

Tali territori considerati minori e ai margini evidenziano non una situazione di ritardo o di arretratezza, ma un nuovo approccio allo sviluppo riconducibile alla qualità e all'abilità del territorio, al di fuori del tradizionale modello fordista, in cui "la ricerca di ritmi differenti e di paesaggi da abitare entro i quali immaginare diversi stili di vita può diventare motivo di agire collettivo, un progetto politicosociale in grado di giustificarsi con i propri risultati e le propria positività" (Lancerini, 2005, p. 17).

Nelle destinazioni minori, in anni recenti, si è attivato un processo orientato a ricostruire nuovamente una identità culturale locale che attribuisce un ruolo importante e integrativo a fattori immateriali quali l'identità locale, le reti, il capitale sociale e la governance (Mutti, 1998; Pichierri, 1995; Trigilia, 1999).

Tali territori sono prevalentemente aree a bassa densità demografica, caratterizzate da un contesto rurale che evidenzia ancora una forte presenza dell'agricoltura, in cui il patrimonio storico-artistico è spesso poco noto, ma di qualità. Il modello di ospitalità che caratterizza tali territori è composto da strutture integrate con il paesaggio locale (bed and breakfast, agriturismo, albergo diffuso) e le attività culturali sono orientate alla valorizzazione delle tradizioni popolari, della storia e dell’identità locale (Calzati, 2011). Si configurano così modelli di sviluppo, diversi da quelli tradizionalmente intesi, in cui si mescolano attività agricole, turismo innovativo e attività produttive originali che evidenziano un'ottica plurisettoriale e quindi l'assenza di un unico motore di sviluppo.

In questo lavoro, non si intende analizzare gli aspetti definitori dello sviluppo sostenibile $^{1}$ con le sue molteplici definizioni (Pearce et al., 1988) che evidenziano forti divisioni e differenze per quanto riguarda gli aspetti applicativi del concetto di sostenibilità, ma affrontare i possibili percorsi di sviluppo di aree definite minori che

1 La definizione di Sviluppo Sostenibile più citata è quella data dalla Commissione Mondiale per l'Ambiente e lo Sviluppo (WCEB), conosciuta come Commissione Bruntland (1987) che definì "sostenibile uno sviluppo che soddisfa le esigenze del presente senza compromettere le possibilità per le generazioni future si soddisfare i propri bisogni" 
individuano nella propria qualità e tipicità ambientale, culturale e paesaggistica il principale elemento di attrazione e competizione.

In anni recenti è il concetto stesso di crescita ad essere messo in discussione in quanto troppo spesso utilizzato come sinonimo di "sviluppo". Il concetto di sviluppo è più ampio e omnicomprensivo di quello di "crescita", in quanto coinvolge aspetti culturali ed etici che lo riconducono a quello di miglioramento di qualità della vita che diventa nelle strategie di sviluppo delle destinazioni minori un possibile fattore competitivo. Quanto esposto implica un cambio di direzione e l'adozione di una valutazione multicriteriale dell'economia in quanto, la natura essenzialmente multidimensionale dello sviluppo sostenibile, comprendente aspetti economici, ambientali, sociali ed istituzionali e richiede quindi l'adozione di misure che rappresentino i vari aspetti di sviluppo sostenibile nel tempo (Shmelev et al., 2009).

Infatti l'approccio alla misurazione della qualità della vita e dello sviluppo sostenibile è cambiato e diviene rilevante il progressivo spostamento a favore di un sistema di valutazione multidimensionale dello sviluppo (Sen, 1999; Layard, 2006; Kahneman e Krueger, 2006, Stiglitz et al., 2009). Inoltre la difficoltà dei misuratori di reddito tradizionali di cogliere i diversi fattori che concorrono alla formazione del benessere possono fare apparire aree arretrate e depresse territori che rappresentano, come afferma Bonomi (2009) in riferimento ai territori montani, un piccolo modello capace di produrre un mix di reddito flessibile e adattivo che sembra tenere rispetto all'impatto con i flussi della crisi.

Solo recentemente con l'apertura del ciclo della green e soft economy, nuova forma di modernità, si è in grado di superare, incorporandole, le tradizionali contrapposizioni imposte dalla modernità stessa: crescita/decrescita, limite/sviluppo. In questo rinnovato contesto le destinazioni minori, attraverso un percorso capace di miscelare forme diverse di innovazione, culturale, sociale, tecnica, di partecipazione e ambientale con una visione condivisa, endogena e comunitaria, possono uscire dai margini e posizionarsi come "distinct areas" orientate a promuovere uno sviluppo territoriale/turistico sostenibile (Calzati, 2011).

\section{Sviluppo turistico e sostenibilità nelle destinazioni minori}

Lo sviluppo economico sostenibile implica la massimizzazione dei benefici netti dello sviluppo economico, a condizione che siano mantenuti nel tempo i servizi e la qualità delle risorse naturali (Pearce et al., 1988). La sostenibilità è un concetto multidimensionale: aspetti economici, sociali ed ambientali devono essere considerati simultaneamente. Infatti in tale contesto, la sostenibilità del turismo se capace di integrare, in un'ottica di coevoluzione, le tre dimensioni fondamentali dello sviluppo, quella ambientale, economica e socio-culturale, può costituire una risposta alla sfida ecologica-economica che i territori sono chiamati a gestire. (Giaoutzi e Nijkamp, 1993; Camagni et al., 2001).

Occorre evidenziare come il termine sostenibilità, nell'ambito degli studi economici e manageriali sul turismo, sia utilizzato in molteplici modi. Si può quindi 
distinguere fra la sostenibilità del turismo e il turismo sostenibile facendo riferimento, nel primo caso, all'approccio adottato dall'UNWTO che attribuisce anche al turismo la logica dell'equilibrio tra le tre dimensioni o pilastri della sostenibilità, coinvolgendo ogni forma di turismo, comprendendo qualsiasi destinazione e includendo sia pratiche turistiche proprie di nicchie di mercato, sia il turismo di massa. Il turismo sostenibile fa riferimento a uno specifico segmento della domanda che ha condotto i vari contributi metodologici ad individuare differenti categorie di segmenti del turismo sostenibile e relativi prodotti. Per quanto riguarda l'analisi della domanda di turismo sostenibile diviene quindi difficile identificare le caratteristiche delle motivazioni e dei comportamenti dei diversi segmenti su cui costruire specifici prodotti, pertanto in letteratura non si hanno definizioni comuni e concordi. Molti autori hanno analizzato e identificato i comportamenti e le motivazioni di turisti con caratteristiche simili individuando gli eco turisti (Fennel, 2002; Bucley, 2003; Weaver e Lawton, 2007), i natur-based tourists (Priskin, 2001; Bucley et al., 2003; Balmford et al., 2009) e i turisti responsabili (Goodwin e Francis, 2003; Colombo, 2005; Sambri e Pegan, 2008; Franch et al., 2008). Sebbene su questo tema in letteratura non si sia giunti a conclusioni univoche, tali segmenti di turismo sostenibile evidenziano alcuni elementi chiave comuni quali: rispetto e salvaguardia dell'ambiente (in particolare ecosistema e biodiversità), riduzione dell'impatto ambientale delle attività legate al turismo, rispetto e salvaguardia della cultura tradizionale delle popolazioni locali. Ulteriori punti di contatto sono la possibile partecipazione attiva delle popolazioni locali nella gestione delle imprese turistiche e la condivisione dei benefici socioeconomici derivanti dal turismo. Queste nuove forme di turismo dovrebbero essere orientate al superamento delle problematiche legate alla scarsa sostenibilità economica, sociale e ambientale tipiche del turismo di massa e promuovere, contestualmente, atteggiamenti e comportamenti pro-ambientali (Fennel, 2001; 2003; Donohe e Needham, 2006). Il passaggio dalle enunciazioni teoriche alle applicazioni pratiche evidenzia, allo stato attuale, contraddizioni che non consentono o rendono difficile proposte turistiche che rispettino i principi base di queste alternative forme di turismo. Infatti il turismo ha un impatto sistemico poiché interessa, con una logica trasversale, l'economia del territorio nel quale si sviluppa (Leiper, 1990; Laws, 1995; Manente e Cerato, 2000; Cooper et al., 2002, Franch, 2010) ed è la logica sistemica a costituire la premessa per la nascita e lo sviluppo di una destinazione (Della Corte, 2009).

Occorre evidenziare inoltre come il turismo sia in grado di apportare trasformazioni ad un territorio sia in termini infrastrutturali, strutturali, sociali ed ambientali (Cooper et al., 2008; Della Corte, 2009; Franch, 2010). Pertanto è necessaria di una gestione del sistema locale al fine di generare valore sostenibile durevole. Fattori quali l'aumento della competizione, l'importanza delle ricadute economiche e sociali che il turismo determina nei territori in cui si sviluppa e le possibili esternalità negative che può produrre, inducono a un approccio di marketing strategico alle destinazioni turistiche. La necessità di definire un processo decisionale di tipo strategico, da parte di un territorio, è considerata una priorità 
orientata a indirizzare i percorsi di sviluppo dell'offerta turistica (Poon, 1993; Phillips e Moutinho, 1998; Vellas e Bècherel, 1999; Godfrey e Clarke, 2000;Ritchie e Crouch, 2000).

Infatti il fenomeno turistico non può essere considerato per i territori un rimedio che produce automaticamente sviluppo locale con significativi risultati nel mediolungo periodo, ma può al contrario innescare meccanismi di impoverimento del territorio, di modifica dell'equilibrio sociale e culturale, di danno alla qualità ambientale, di esigenze di consumo sproporzionate rispetto a quelle abituali e accettabili per la destinazione (McKercher, 1993; Hunter e Green, 1995).

La sostenibilità del modello di sviluppo è essa stessa un valore capace di agire sulla competitività della destinazione perciò esiste un legame stretto tra sostenibilità e competitività turistica (Middleton e Hawkins, 1998; Mihalic, 2000) in quanto la tutela e conservazione dell'ambiente e l'equilibrio sociale interno concorrono a migliorare la qualità dell'offerta turistica e a generare ricadute economiche di medio e lungo periodo e perciò non devono essere considerati limiti, ma condizioni per lo sviluppo.

La questione principale riguarda la sostenibilità della destinazione, come misurarla e quindi come stabilire se essa è sostenibile. Nel 2004 il WTO ha pubblicato la Guida degli indicatori di sviluppo sostenibile per le destinazioni turistiche in cui si individuano vari indicatori e si propone agli amministratori e agli attori delle destinazioni una metodologia per sviluppare indicatori nel contesto dei processi di pianificazione turistica.

Il conseguimento dell'obiettivo di sostenibilità dipende, dunque, dalla capacità dei diversi soggetti coinvolti di coordinare le proprie scelte di sfruttamento della risorsa ambientale, nei limiti della sua fruizione sostenibile. I territori minori si configurano come destinazioni di tipo community (Murphy, 1985; Kaspar, 1995; Martini, 2005), cioè luoghi che attraverso l'interazione delle attività dei molti attori locali si posizionano sul mercato turistico con uno specifico brand. Occorre evidenziare come nelle destinazioni community il principale problema è costituito dal coordinamento tra gli attori, che non può essere incentivato attraverso meccanismi di coordinamento di tipo gerarchico, ma analizzato utilizzando gli schemi concettuali della teoria delle reti o della stakeholder theory (Robson e Robson, 1996; Sautter e Leisen, 1999; Bramwell e Sharman, 1999; Della Corte, 2000; Martini, 2005).

Le destinazioni minori caratterizzate da risorse naturali e culturali difficilmente riproducibili altrove e capaci di caratterizzare un'offerta turistica fortemente identitaria, riescono a promuovere modelli di sviluppo endogeno in cui il coinvolgimento diretto della comunità locale esercita, ed è garanzia, di un maggiore controllo sulle dimensione della sostenibilità ecologica e sociale soprattutto se i modelli decisionali sono di tipo partecipativo (Simmons, 1994; Timothy, 1999).

A differenza dei grandi sistemi metropolitani che, a fronte di forti vantaggi competitivi in termini economici, evidenziano problematiche connesse con l'equità sociale ed ambientale, le destinazioni minori riescono a perseguire percorsi di coevoluzione delle tre aree della sostenibilità (Ciciotti et. al., 2008), promuovendo 
modelli di sviluppo turistico sostenibile considerati eccellenti. È questa la finalità del progetto comunitario EDEN rivolto a quelle destinazioni che meglio hanno integrato sviluppo ambientale, crescita economica e coesione sociale costituendo buone pratiche da diffondere a livello comunitario.

\section{La sostenibilità delle destinazioni nella politica europea per il turismo}

Ai fini del presente lavoro si è ritenuto importante un'analisi della Comunicazione della Commissione Europea " $L$ 'Europa, la destinazione turistica $n$. 1 del mondo. Un nuovo quadro di riferimento politico per il turismo in Europa (2010) in cui si evidenzia il ruolo strategico che il turismo riveste nel contesto europeo e la necessità di definire un nuovo quadro d'azione delle iniziative in materia di turismo, per rafforzarne la competitività e la sua capacità di crescita sostenibile, implementando così il processo di definizione della politica europea per il turismo.

Il nuovo piano di azione della Commissione rappresenta un sostegno alle sfide che attualmente determinano un forte impatto sulla domanda turistica europea e che impongono l'adozione di misure sempre più rivolte ad un consumo turistico sostenibile e responsabile. Si intende favorire un approccio coordinato alle iniziative in materia di turismo e definire un nuovo quadro d'azione che individua nel turismo uno strumento capace di rafforzare l'immagine dell'Europa nel mondo, di diffonderne i valori e promuovere l'interesse per il modello europeo che ha origine da secoli di scambi culturali, diversità linguistica e creatività.

Nel documento si evidenziano, in linea con i principi del trattato di Lisbona ${ }^{2}$, gli obiettivi della politica europea in tema di turismo. Gli obiettivi sono quattro:

1) stimolare la competitività del settore turistico in Europa;

2) promuovere lo sviluppo del turismo sostenibile, responsabile e di qualità;

3) consolidare l'immagine e la visibilità dell'Europa come insieme di destinazioni sostenibili di qualità;

4) massimizzare il potenziale delle politiche e gli strumenti finanziari dell'UE per lo sviluppo del turismo.

Gli obiettivi e le azioni previste dal documento indicano come la politica europea del turismo mira principalmente a stimolare la competitività del settore, senza dimenticare che, a lungo termine, la competitività è strettamente connessa alla sostenibilità del suo modello di sviluppo.

Non è obiettivo del presente lavoro l'analisi delle 21 azioni previste, tuttavia particolare attenzione è rivolta all'obiettivo Promuovere lo sviluppo di un turismo sostenibile, responsabile e di qualità, in quanto sono presenti i temi oggetto di tale studio: il legame sostenibilità/competitività; la necessità di un sistema di indicatori

2 Nel Trattato di Lisbona (2007) il turismo è riconosciuto come settore strategico per l'economia europea, grazie alla sua elevata capacità di produrre crescita e occupazione. 
utile per la gestione sostenibile di una destinazione e lo sviluppo di un marchio come strumento di promozione delle destinazioni. Dall'analisi delle azioni previste si evidenzia la necessità di elaborare un sistema di indicatori per la gestione sostenibile delle destinazioni partendo dalle esperienze degli enti regionali e dalle competenze delle reti NECSTouR e EDEN; di creare un marchio europeo "Turismo di qualità" finalizzato da un lato a stimolare le destinazioni ad applicare pratiche sostenibili e dall'altro a rafforzare l'immagine dell'Europa come destinazione turistica di qualità. Il brand management applicato al territorio (Blain et al., 2005; Kavaratzis, 2005; Martini, 2005; Golinelli e Simoni; 2006) ha origine dal concetto di brand value adottato per le imprese (Golinelli, 2004). Il brand territoriale si differenzia dal brand aziendale in quanto fregia un intero territorio caratterizzato per la presenza di risorse naturali e artificiali e per una molteplicità di attori coinvolti nella definizione dell'offerta turistica. Esso esprime un'immagine e un'identità della destinazione condivisa dagli attori privati e dagli operatori pubblici e percepita come unica dall'esterno (Franch et al., 2008). La creazione di un brand territoriale, svolge un ruolo importante nella politica di comunicazione turistica influendo sull'immagine e sulla notorietà della destinazione, elementi alla base della sua capacità di attrazione e della sua competitività (Pechlaner et al., 2005; Peters et al., 2006). Esso diviene possibile strumento capace di accrescere la competitività del territorio, di creare una identità condivisa e di rafforzare la collaborazione tra enti pubblici e stakeholder privati.

Si sottolinea inoltre il ruolo del fattore qualità nella competitività tra destinazioni turistiche e come l'incremento della qualità di tutti i servizi, risulti essere fondamentale, al fine di dotare le destinazioni turistiche europee di un maggiore vantaggio competitivo. La promozione di modelli di turismo sostenibile diventa un percorso realizzabile solo in un'ottica di cooperazione e condivisione pertanto si richiede una stretta collaborazione tra l'Unione Europea e i principali mercati esistenti e i Paesi vicini in particolare i Paesi del Mediterraneo. Il nuovo quadro d'azione oltre a incrementare la competitività e la crescita sostenibile del turismo intende rafforzare l'identità europea e la diffusione del senso di appartenenza ad una comune cittadinanza garantendo il rispetto reciproco, il dialogo interculturale e lo scambio di conoscenze.

\section{II progetto EDEN (European Destinations of Excellence) nella Regione Lombardia}

\subsection{Progetto EDEN: origini e obiettivi}

Il Progetto Destinazioni Europee di Eccellenza (o EDEN), acronimo di European Destinations of ExcelleNce), è un'iniziativa promossa dalla Commissione Europea al fine di promuovere in tutta l'Unione Europea modelli di sviluppo turistico sostenibile. Il progetto nasce nel 2006 e prevede l'assegnazione di un riconoscimento, attraverso un concorso annuale, alle destinazioni minori, non 
coinvolte dal turismo di massa, che mirano a raggiungere obiettivi di crescita economica e sviluppo turistico in un'ottica di sostenibilità ambientale, sociale e culturale.

Come già evidenziato il turismo rappresenta un'attività economica strategica nell'Unione Europea la cui importanza è destinata ad aumentare nei prossimi anni. Il turismo costituisce uno strumento in grado di contribuire alla realizzazione di diversi obiettivi dell'UE quali lo sviluppo sostenibile, la crescita economica, l'occupazione e la coesione sociale. In questo contesto si inserisce il progetto EDEN come iniziativa in grado di promuovere e dare visibilità a destinazioni minori capaci, da un lato, di fare sistema al fine di superare una connotazione settoriale che ha limitato le potenzialità di sviluppo del turismo italiano e dall'altro di coinvolgere la comunità locale stabilendo una specie di patto sociale che nel promuovere l'accoglienza del turista contestualmente promuove la qualità della vita dei residenti. Gli obiettivi di lungo periodo del progetto riguardano: il rafforzamento della visibilità delle emergenti destinazioni turistiche Europee d'eccellenza; la creazione di una piattaforma per la condivisione di buone pratiche in tutto il territorio UE; la premiazione e il sostegno di forme di turismo sostenibile. Inoltre il progetto intende favorire il decongestionamento delle destinazioni turistiche più visitate e trasformare le località meno note in destinazioni turistiche per tutto l'anno; stimolare l'attenzione sul valore, la diversità e le caratteristiche comuni delle destinazioni europee e promuovere la creazioni di reti tra le destinazioni premiate finalizzate a incentivare in altre destinazioni l'adozione di modelli sostenibili di sviluppo turistico.

Il progetto EDEN rappresenta quindi un'opportunità di visibilità internazionale per le località minori; di scambio di buone pratiche tra i Paesi membri e di stimolo a valorizzare principalmente il patrimonio immateriale locale sia per i residenti che per i turisti in una visione di sviluppo turistico endogeno, sostenibile e durevole.

\subsection{Il processo e i criteri di selezione}

Il progetto prevede, attraverso un concorso annuale, la selezione in ogni Stato membro di destinazioni proposte come esempio di buone pratiche di sviluppo turistico sostenibile. La competizione si svolge su scala nazionale, con il coinvolgimento delle Amministrazioni centrali degli Stati membri e candidati (Ministeri, Enti governativi, ecc.), i quali hanno il compito di individuare nel proprio territorio 5 destinazioni finaliste tra le quali verrà poi scelta la vincitrice. Ogni anno la Commissione Europea congiuntamente agli organismi competenti del turismo nazionale individua un tema che costituisce il filo conduttore dell'iniziativa ed è finalizzato a dare visibilità ai diversi aspetti delle risorse delle regioni europee ed è collegato allo sviluppo sostenibile in termini economici, ambientali, culturali e di coinvolgimento della comunità locale. Le destinazioni vincitrici sono quelle che meglio rispondono al tema scelto e che offrono un'esperienza turistica unica coerente con i criteri di sostenibilità. I temi individuati nelle precedenti edizioni sono: 
- turismo rurale (2007),

- turismo e patrimonio immateriale locale (2008),

- turismo e aree protette (2009),

- turismo acquatico (2010),

- turismo e riconversione dei siti (2011),

- turismo accessibile (2012-2013).

Le destinazioni per poter partecipare devono rispondere a due criteri di selezione: quelli stabiliti dalla Commissione Europea e quelli individuati dai singoli Comitati Nazionali di Valutazione. I primi che hanno carattere generale sono:

a) essere una destinazione "non tradizionale", con una bassa o molto bassa densità di turisti;

b) organizzare con una periodicità regolare un evento specifico legato al proprio patrimonio immateriale;

c) gestire la propria offerta turistica in modo tale da assicurare la sostenibilità sociale, culturale e ambientale.

I secondi, più dettagliati, sono stabiliti dal Comitato Nazionale di Valutazione istituito in ogni Paese partecipante. In Italia è presieduto dal Dipartimento per lo Sviluppo e la Competitività del Turismo della Presidenza del Consiglio dei Ministri, e supportato da un'apposita Segreteria tecnica. Del Comitato fanno parte i rappresentanti del Ministero dell'Ambiente e Tutela del Territorio e del Mare, delle Regioni, attraverso il Coordinamento Regionale per il Turismo, dell'ENIT - Agenzia Nazionale del Turismo, dell' ANCI - Associazione Nazionale dei Comuni Italiani e dell'UNCEM - Unione Nazionale Comuni, Comunità, Enti montani.

Gli aspetti che dovranno essere esaminati ai fini della valutazione per la scelta della destinazione sono:

- valorizzazione del patrimonio culturale immateriale locale presso i residenti e i turisti;

- azioni innovative intese a promuovere le risorse immateriali locali e attenzione per il turista;

- coinvolgimento delle piccole imprese locali e dei residenti ed eventuali finanziamenti;

- qualità dell'offerta turistica basata sulle risorse immateriali e integrazione degli aspetti sociali economici ed ambientali;

- ricettività e servizi;

- accesso alla località.

Occorre evidenziare l'importanza attribuita in fase di selezione alle risorse immateriali locali, alla qualità e al coinvolgimento della comunità locale: fattori che come evidenziato sono capaci di creare valore aggiunto per una destinazione, in quanto non riproducibili altrove e capaci quindi di offrire un'esperienza turistica unica e irripetibile. 


\subsection{Il progetto EDEN: il caso della Regione Lombardia}

La Regione Lombardia, nella lista delle destinazioni italiane selezionate dalla prima edizione dell'iniziativa (2007) ad oggi, è presente nel 2009 con la Riserva Naturale Valli del Mincio posizionatasi al $3^{\circ}$ posto e nel 2010 con il comune di Monte Isola (BS) classificatosi al $1^{\circ}$ posto (tabella 1 ).

L'obiettivo di questa parte del lavoro è stato quello di descrivere e interpretare l'esperienza lombarda, attraverso la raccolta e l'analisi delle testimonianze degli attori promotori/referenti della candidatura della destinazione e della documentazione resa disponibile dal Dipartimento per lo sviluppo e la competitività del turismo, dal Comune di Monte Isola e dal Parco del Mincio.

In particolare sono state analizzate le schede di candidatura presentate dal Comune di Monte Isola e dal Parco del Mincio e realizzate interviste semistrutturate ai rispettivi responsabili del progetto. L'obiettivo è stato quello di ricostruire un quadro delle esperienze, evidenziando le potenzialità e le criticità di questo strumento.

Tab. 1: Destinazioni EDEN Regione Lombardia

\begin{tabular}{|l|l|l|l|l|l|l|}
\hline $\begin{array}{l}\text { Destinazione } \\
\text { Candidata }\end{array}$ & $\begin{array}{l}\text { Soggetto } \\
\text { proponente }\end{array}$ & Anno & Tema & Residenti & $\begin{array}{l}\text { Strutture } \\
\text { Ricettive }\end{array}$ & $\begin{array}{l}\mathbf{N}^{\circ} \text { di } \\
\text { arrivi }\end{array}$ \\
\hline $\begin{array}{l}\text { Riserva } \\
\text { Naturale Valli } \\
\text { del Mincio }\end{array}$ & $\begin{array}{l}\text { Parco del } \\
\text { Mincio }\end{array}$ & 2009 & $\begin{array}{l}\text { "Turismo e } \\
\text { Aree protette" }\end{array}$ & $18.612\left(^{*}\right)$ & $22\left(^{*}\right)$ & $5.582\left(^{*}\right)$ \\
\hline $\begin{array}{l}\text { Comune di } \\
\text { Monte Isola } \\
\text { (BS) }\end{array}$ & $\begin{array}{l}\text { Comune di } \\
\text { Monte Isola } \\
\text { (BS) }\end{array}$ & 2010 & $\begin{array}{l}\text { "Turismo } \\
\text { Acquatico" }\end{array}$ & $1.811\left(^{* *}\right)$ & $6\left(^{* *}\right)$ & $500\left(^{* *}\right)$ \\
\hline
\end{tabular}

$\left(^{*}\right)$ dati relativi all'anno 2007

$\left({ }^{* *}\right)$ dati relativi all'anno 2009

Fonte: ns elaborazione su dati del Comune di Monte Isola e del Parco Regionale del Mincio

Monte Isola è l'isola lacustre più vasta d'Italia con una superficie di 4,5 $\mathrm{km}^{2}$ e un perimetro di oltre $9 \mathrm{~km}$ è la prima in Europa come altezza sul livello del mare, raggiungendo un'altitudine di 600 metri.

La Riserva Naturale "Valli del Mincio" è costituita da un territorio compreso nei comuni di Rodigo, Porto Mantovano, Curtatone e Mantova. Il sistema palustre che si estende su una superficie di circa 1100 ettari, interessando un tratto di fiume di 7-8 $\mathrm{km}$ rappresenta la più estesa palude interna italiana dichiarata Riserva Naturale dalla Regione Lombardia nel 1984 fa parte della Rete Natura $2000^{3}$.

3 Natura 2000 è il principale strumento della politica dell'Unione Europea per la conservazione della biodiversità. Si tratta di una rete ecologica diffusa su tutto il territorio dell'Unione, istituita ai sensi della Direttiva 92/43/CEE "Habitat" al fine del mantenimento a lungo termine degli habitat naturali e delle specie di flora e fauna minacciati o rari a livello comunitario. La rete Natura 2000 comprende Zone Speciali di Conservazione (ZSC) istituite dagli Stati Membri secondo quanto stabilito dalla Direttiva 
Entrambi i territori presentano le caratteristiche proprie delle destinazioni minori e quindi di quelle aree definite nella prima parte del lavoro ai margini del turismo di massa, ma caratterizzate da ottima qualità ambientale, presenza di patrimonio culturale anche se poco noto, da un modello di ospitalità composto da strutture integrate con il paesaggio locale e da attività culturali finalizzate alla valorizzazione delle tradizioni popolari, della storia e dell'identità locale.

Le tabelle 2 e 3 evidenziano, in sintesi, le azioni realizzate dalle due destinazioni lombarde per attivare un percorso di sviluppo turistico sostenibile che individua nel partenariato pubblico-privato un possibile strumento di gestione del territorio.

Numerosi sono gli studi sul turismo (Fayos e Sola, 1996; Bramwell e Sharman, 1999; Bramwell e Lane, 2000; Pfoor, 2002; Dredge, 2006; Scott et al., 2008; Beritelli, 2010; Haugland et al., 2011) che evidenziano come la cooperazione giochi un ruolo centrale nello sviluppo delle destinazioni turistiche, come il turismo si caratterizzi per il coinvolgimento di diversi livelli decisionali territoriali e per una numerosità di soggetti interessati.

Tab. 2: Azioni realizzate e presenza di partenariati pubblico-privato

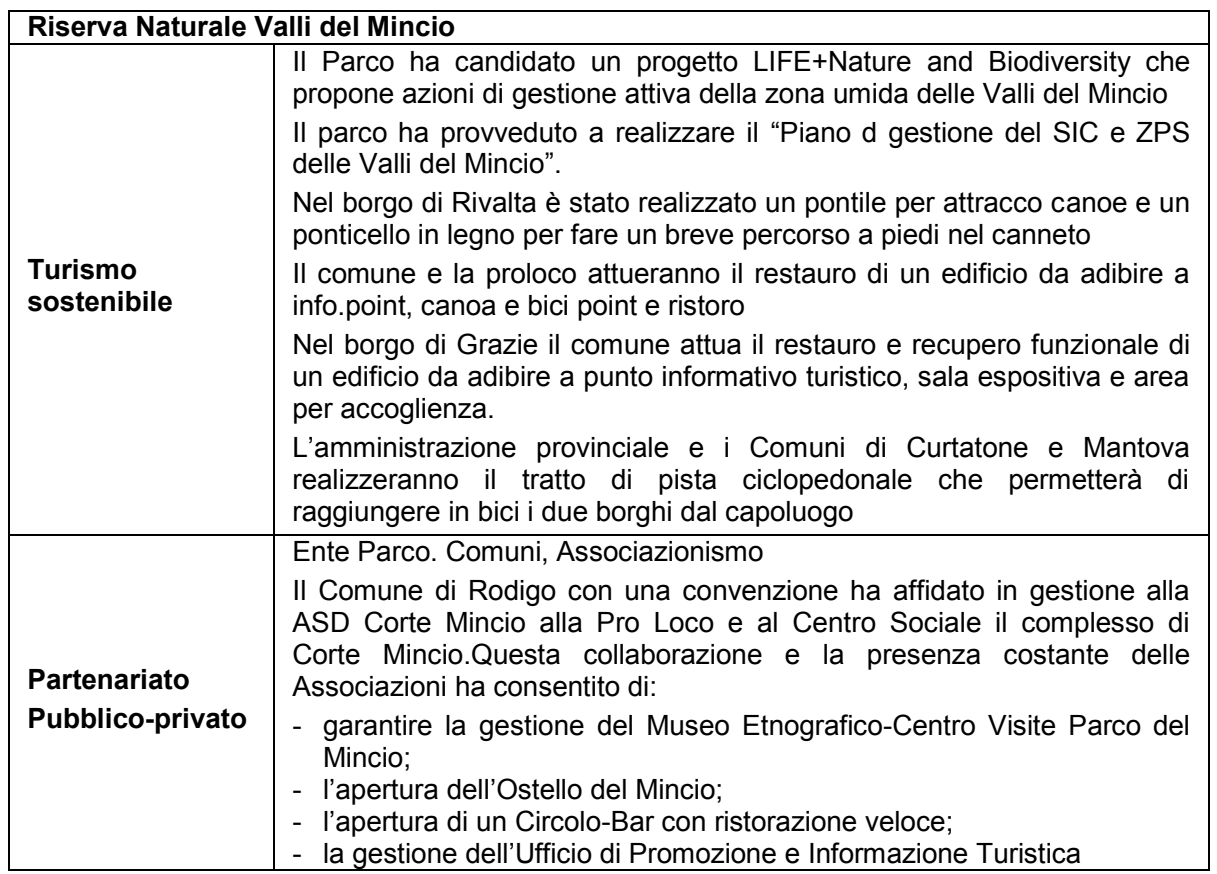

Fonte: ns elaborazione su dati del Parco Regionale del Mincio

Habitat, e le Zone di Protezione Speciale (ZPS) istituite ai sensi della Direttiva 79/409/CEE "Uccelli". 
Tab. 3: Azioni realizzate e presenza di partenariati pubblico-privato

\begin{tabular}{|l|l|}
\hline Comune di Monte Isola (BS) \\
\hline Turismo sostenibile & $\begin{array}{l}\text { La variante generale approvata dal PRG nel } 2004 \text { ha posto limiti ben precisi, al fine di } \\
\text { limitare la trasformazione del paesaggio e garantire il permanere di testimonianze } \\
\text { storiche, fissando precisi criteri e modalità di intervento nelle varie zone. } \\
\text { Realizzazione di un percorso Didattico - Naturalistico denominato "Sentiero delle } \\
\text { Rocca". } \\
\text { II recupero delle vecchie mulattiere e dei sentieri: attraverso la collaborazione con il } \\
\text { consorzio forestale del Sebino e la Comunità Montana del Sebino Bresciano }\end{array}$ \\
\hline $\begin{array}{l}\text { Reti associative } \\
\text { Partenariato } \\
\text { Pubblico-privato }\end{array}$ & $\begin{array}{l}\text { Associazione Nazionale I "Borghi più Belli d'Italia } \\
\text { Comunità Montana degli Ulivi }\end{array}$ \\
& $\begin{array}{l}\text { Piano integrato d'area del medio lago (Comuni di Marone, Monte Isola, Sale Marasino, } \\
\text { Sulzano) } \\
\text { Progetto Bike sharing (CM del Sebino Bresciano e Navigazione lago d'Iseo s.r.I.) } \\
\text { finanziato dalla Fondazione Cariplo }\end{array}$ \\
\hline
\end{tabular}

Fonte: ns elaborazione su dati del Comune di Monte Isola

Le interviste ai responsabili del progetto EDEN nelle due destinazioni ha evidenziato l'impegno degli amministratori locali di caratterizzare il proprio territorio come area ad alta qualità ambientale, paesaggistica ed enogastronomica al fine di salvaguardare la valorizzazione della cultura e delle tradizioni locali nonché la qualità dell'ospitalità turistica al fine di promuovere sviluppo turistico sostenibile.

Le interviste hanno riguardato nello specifico l'individuazione dei punti di forza e di debolezza del territorio, le motivazioni alla base della candidatura gli scambi di buone pratiche e i vantaggi e le ricadute a seguito del riconoscimento. (Tabella $4 \mathrm{e}$ $5)$.

Tab. 4: Sintesi risultati intervista al responsabile Servizio Comunicazione ed Educazione ambientale Parco Regionale del Mincio

\begin{tabular}{|c|c|c|c|c|c|c|}
\hline \multicolumn{7}{|c|}{ Parco del Mincio } \\
\hline $\begin{array}{c}\text { Anno di } \\
\text { partecipazione }\end{array}$ & Tema & $\begin{array}{c}\text { Punti di forza del } \\
\text { Territorio } \\
\end{array}$ & $\begin{array}{c}\text { Punti di debolezza } \\
\text { del territorio }\end{array}$ & $\begin{array}{c}\text { Perché } \\
\text { candidarsi }\end{array}$ & $\begin{array}{c}\text { Scambi buone } \\
\text { pratiche }\end{array}$ & $\begin{array}{c}\text { Vantaggi - } \\
\text { ricadute }\end{array}$ \\
\hline 2008-2009 & $\begin{array}{l}\text { Turismo } \\
\text { ed aree } \\
\text { protette }\end{array}$ & 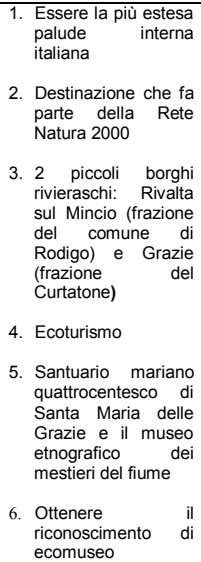 & $\begin{array}{l}\text { 1. Risorse } \\
\text { economiche } \\
\text { limitate } \\
\text { 2. Incapacità di } \\
\text { fare rete } \\
\text { 3. Scarsa } \\
\text { attenzione al } \\
\text { patrimonio } \\
\text { ambientale } \\
\text { 4. Abbandono } \\
\text { della pratica di } \\
\text { coltivazione dei } \\
\text { canneti }\end{array}$ & $\begin{array}{l}\text { II territorio } \\
\text { possiede i } \\
\text { requisiti } \\
\text { richiesti dal } \\
\text { bando }\end{array}$ & $\begin{array}{l}\text { Non realizzate a } \\
\text { causa di limiti } \\
\text { operativi interni } \\
\text { e della } \\
\text { mancanza di } \\
\text { una rete attiva } \\
\text { tra lestinazioni } \\
\text { Eden } \\
\text { Ese }\end{array}$ & $\begin{array}{l}\text { 1. } \begin{array}{l}\text { Nessun } \\
\text { vantaggio } \\
\text { concreto }\end{array} \\
\text { 2. Aumento } \\
\text { della } \\
\text { visibilità sui } \\
\text { media } \\
\text { locali }\end{array}$ \\
\hline
\end{tabular}

Fonte: ns elaborazione su dati del Parco Regionale del Mincio 
Tab. 5: Sintesi risultati intervista al sindaco del Comune di Monte Isola

\begin{tabular}{|c|c|c|c|c|c|c|}
\hline \multicolumn{7}{|c|}{ Comune di Monte Isola (BS) } \\
\hline $\begin{array}{c}\text { Anno di } \\
\text { partecipazion } \\
\text { e }\end{array}$ & Tema & $\begin{array}{l}\text { Punti di forza del } \\
\text { Territorio } \\
\end{array}$ & $\begin{array}{c}\text { Punti di } \\
\text { debolezza del } \\
\text { Territorio } \\
\end{array}$ & Perché candidarsi & $\begin{array}{c}\text { Scambi } \\
\text { buone } \\
\text { pratiche }\end{array}$ & $\begin{array}{l}\text { Vantaggi - } \\
\text { ricadute }\end{array}$ \\
\hline 2010 & $\begin{array}{l}\text { Turismo } \\
\text { Acquatico }\end{array}$ & $\begin{array}{l}\text { 1. Essere l'sola } \\
\text { lacustre abitata } \\
\text { più grande } \\
\text { d'Europa. } \\
\text { 2. } 2 \text { isolette di } \\
\text { San Paolo e di } \\
\text { Loreto } \\
\text { 3. } 6 \text { chiese } \\
1 \text { museo } \\
1 \text { castello } \\
\text { 4. Naet } \\
\text { imbarcazione } \\
\text { simbolo del } \\
\text { lago d'lseo } \\
\text { (cantieri nautici) }\end{array}$ & $\begin{array}{ll}\text { 1. } & \begin{array}{l}\text { Morfologia } \\
\text { del territorio }\end{array} \\
\text { 2. } & \text { Limitazione } \\
\text { nei trasporti }\end{array}$ & $\begin{array}{l}\text { 1. L'Amministra- } \\
\text { zione ha } \\
\text { operato da } \\
\text { sempre scelte } \\
\text { precise per } \\
\text { l'accoglienza } \\
\text { turistica via } \\
\text { acqua } \\
\text { 2. II comune } \\
\text { presenta le } \\
\text { caratteristiche } \\
\text { richieste dal } \\
\text { bando }\end{array}$ & $\begin{array}{l}\text { Creazione, } \\
\text { tuttora in } \\
\text { corso, con le } \\
\text { destinazioni } \\
\text { europee } \\
\text { vincenti } \\
\text { dell'Associazio } \\
\text { ne EDEN che } \\
\text { ha come } \\
\text { finalità lo } \\
\text { scambio di } \\
\text { buone pratiche } \\
\text { all'interno della } \\
\text { rete EDEN }\end{array}$ & $\begin{array}{l}\text { Aumento della } \\
\text { visibilità a } \\
\text { livello } \\
\text { nazionale e } \\
\text { internazionale }\end{array}$ \\
\hline
\end{tabular}

Fonte: ns elaborazione su dati del Comune di Monte Isola

Il punto di forza di entrambi i territori è la qualità ambientale a conferma di una visione del territorio in cui al centro vi è sempre di più l'abitabilità e la qualità, in una prospettiva di sviluppo sostenibile che molto bene si coniuga con il paesaggio sostenibile, cioè una valutazione del paesaggio come indicatore veritiero di sostenibilità o insostenibilità (Manzi, 2001). A conferma di quanto precedentemente esposto le destinazioni oggetto d'indagine sono in grado di rappresentare proposte turistiche che rispettino i principi base di alternative forme di turismo quali l'ecoturismo considerato dagli intervistati una opportunità per il territorio. I punti di debolezza per quanto riguarda Monte Isola sono riconducibili alla limitazione nei trasporti causata dalla morfologia del territorio. La Riserva "Valli del Mincio" individua nella scarsa disponibilità di risorse economiche e nella difficoltà della comunità locale a "fare rete" le principali criticità. Il possesso dei requisiti richiesti dal bando è la principale motivazione alla base della candidatura delle due destinazioni oggetto d'indagine.

Occorre evidenziare come entrambe i responsabili del progetto individuano nell'aumento della visibilità e della notorietà della destinazione il principale vantaggio ottenuto dal riconoscimento. A tal fine si sottolinea che non sono utilizzati indicatori mirati a valutare il reale impatto di questo incremento.

Evidenziano, inoltre, come non si sia realizzato quello scambio di buone pratiche tra le destinazioni vincitrici che rappresenta uno degli obiettivi del progetto comunitario, anche se il sindaco di Monte Isola ha sottolineato come la recente costituzione tra le destinazioni vincitrici europee di una "Associazione Eden" finalizzata allo scambio di buone pratiche all'interno della rete EDEN, può costituire un primo step in questa direzione. 


\section{Conclusioni}

Le brevi riflessioni sulla base dell'attività svolta evidenziano come i territori minori, caratterizzati da produzioni di eccellenza, dalla bellezza del patrimonio storico-artistico e del paesaggio e dalla qualità dell'offerta locale, possano dare origine all'interno dei territori regionali a delle "distinct areas" in cui lo sviluppo turistico è il risultato di una nuova esperienza di fruizione del territorio. Alcuni di questi territori, turisticamente definiti come destinazioni minori, escluse dai circuiti del turismo di massa, non sono aree marginali con un'offerta turistica qualitativamente debole o assente e quindi incapaci di promuovere sviluppo turistico, ma sono destinazioni premiate come eccellenti sulla base di una marginalità che assume connotazione positiva. Infatti il legame tra sviluppo locale e marginalità è interpretato come espressione di opportunità e attraverso la valorizzazione delle risorse locali materiali e immateriali in cui il capitale sociale, inteso come fiducia, reciprocità, condivisione di valori collettivi (Putnam, 1993; Fukuyama, 1995, 1996; Mutti, 1998; Trigilia, 1999), consente agli attori locali di giocare un ruolo sulle traiettorie di sviluppo sostenibile.

Dall'analisi del caso emergono due considerazioni importanti, la prima individua nel progetto comunitario EDEN uno strumento capace di aumentare la visibilità e la notorietà di una destinazione minore, la seconda evidenzia l'inadeguatezza dello stesso nel creare una piattaforma per la condivisione e lo scambio di buone pratiche come previsto negli obiettivi. Per quanto riguarda la prima considerazione sarebbe utile la costruzione di indicatori mirati a comprendere se vi sia stato un reale impatto sulla comunicazione turistica. Per quanto riguarda invece la seconda considerazione, forse la realizzazione di uno strumento capace di promuovere la diffusione di buone pratiche, non solo tra le destinazioni vincitrici ma anche tra le altre destinazioni minori UE, necessita di maggior tempo.

Il lavoro, costituisce una prima riflessione, sulle potenzialità di sviluppo turistico delle destinazioni minori, in una visione in cui la marginalità di tale aree è fattore distintivo di sviluppo e sulla capacità di questi territori di perseguire percorsi di coevoluzione delle tre aree della sostenibilità in grado di promuovere modelli di sviluppo turistico sostenibile, considerati eccellenti e, pertanto, buone pratiche da diffondere a livello comunitario.

Infine la ridotta estensione numerica dei casi analizzati costituisce un limite del lavoro, pertanto sarebbe opportuno ampliare l'analisi a tutte le destinazioni EDEN italiane, al fine di delineare ulteriori opportunità, evidenziare criticità di questo strumento e le azioni di sviluppo sostenibile che caratterizzano le destinazioni minori ritenute eccellenti. Ulteriore tema di approfondimento futuro riguarda la governance delle attività di branding territoriale, in quanto se le azioni dei singoli attori non risultassero coordinate tra loro ci sarebbe la presenza di messaggi incoerenti e la definizione dell'immagine territoriale risulterebbe non solida e la reputazione della destinazione impossibile da indirizzare (Cantone et al., 2007; Golinelli et al., 2006; Anholt, 2007) rendendo così inutile per il territorio l'ottenimento del premio. 


\section{Bibliografia}

ANHOLT S. (2007), L'identità competitiva, Egea, Milano.

BALMFORD A., BERESFORD J., GREEN J., NAIDOO R., WALPOLE M., MANICA A. (2009), A Global Perspective on Trends in Nature-Based Tourism, PLoS Biol, vol.7, Isue 6, pp.1-6, e1000144. doi:10.1371/journal.pbio.1000144.

BERITELLI P. (2010), Cooperation among prominent actors in a tourist destination, Annals of Tourism Research, vol. 38, n. 2, pp. 607-629.

BLAIN C., LEVY S.E., RITCHIE J.R.B. (2005), "Destination Branding", Journal of Travel Research, vol. 43, n. 4, pp. 328-338.

BONOMI A., (2009), "La piattaforma alpina nell'ipermodernità", in Borghi E., La sfida dei territori nella green economy, Roma, Il Mulino.

BONOMI A., RULLANI E. (2005), Il capitalismo personale. Vite al lavoro, Einaudi, Torino.

BRAMWELL B., SHARMAN A. (1999), "Collaboration in local tourism policy making", Annals of Tourism Research, vol. 26, n. 2, pp. 392-415.

BRAMWELL B., LANE B. (2000), Tourism collaboration and partnership, politics, practice and sustainability, Channel View publications, Clevedon.

BUCKLEY R. (2003), Case Studies in Ecotourism, Cabi, Wallingford.

BUCKLEY R., PICKERING C., WEAVER D.B. (2003), Nature-based Tourism, Environment and Land Management, Cabi, Wallingford.

CALZATI V., (2011), "Territori lenti: nuove traiettorie di sviluppo" in Calzati V., De Salvo P., Nocifora E. (a cura di), Territori lenti e turismo di qualità. Prospettive innovative per lo sviluppo di un turismo di un turismo di qualità, Franco Angeli, Milano.

CAMAGNI R., CAPELLO R., NIJKAMP P. (2001), "Managing sustainable urban environments", in Paddison R. (a cura di), Handbook of urban studies, Sage, Londra.

CANTONE L., RISITANO M., TESTA P. (2007), "Strategie di sviluppo delle destinazioni turistiche e ruolo della marca territoriale", Mercati e Competitività, n. 1., pp.23-32

CICIOTTI E., DALLARA A., RIZZI P. (2008), Una geografia della sostenibilità dei sistemi locali italiani, Internazionalizzazione e sviluppo regionale, Franco Angeli, Milano.

COLOMBO L. (2005), Il turismo responsabile, Xenia Edizioni, Milano.

COMMISSIONE EUROPEA (2010), Comunicazione 352/3, L'Europa, prima destinazione turistica mondiale - un nuovo quadro politico per il turismo europeo.

COOPER C., FLETCHER J., FYALL A., GILBERT D., WANHILL S., (2008), Tourism: Principles and practice, Harlow, FT Prentice Ha.

COOPER C., FLETCHER J., GILBERT D., SHEPHERD R., WANHILL S., (2002), Economia del turismo, Zanichelli, Bologna.

DELLA CORTE V. (2009), Imprese e sistemi turistici. Il management, Egea, Milano

DELLA CORTE V. (2000), La gestione dei sistemi locali di offerta turistica, Cedam, Padova.

DONOHOE H.M., NEEDHAM R.D., (2006), "Ecotourism: The evolving contemporary definition", Journal of Ecotourism, vol. 5, n. 3, pp. 192-210.

DREDGE D. (2006), "Policy networks and the Local Organisation of Tourism", Tourism Management, vol. 27, n. 2, pp. 269-280.

FAYOS-SOLA E. (1996), “Tourism policy: a midsummer night's dream?", Tourism Management, vol. 17, n. 6, pp. 405-412.

FENNEL D.A. (2001), "A content analysis of ecotourism definitions", Current Issues in Tourism, vol. 4, n. 5, pp. 403-421.

FENNEL D.A. (2002), Ecotourism Programme Planning, Cabi, Wallingford.

FENNEL D.A., DOWLING R.K., (2003), Ecotourism policy and planning, CABI Publishing, Wallingford, Oxon. 
FRANCH M., SAMBRI C., MARTINI U., PEGAN G., RIZZI G. (2008), "La domanda di turismo responsabile e di eco-turismo in Italia. Un'indagine esplorativa sui turisti CTS”, Atti del Convegno Marketing Trends, Università Ca' Foscari Venezia, 17-19 gennaio 2008.

FRANCH M. (2010), Marketing delle destinazioni turistiche. Metodi, approcci e strumenti, McGraw-Hill, Milano.

FRANCH M., MARTINI U., BUFFA F. (2008), "Strategie di brand management nelle destinazioni alpine community", Mercati e Competitività, n. 4 pp. 151-170.

FUKUYAMA F. (1995), Trust. The Social Virtues and the Creation of Prosperity, FreePress, New York.

FUKUYAMA F. (1996), Fiducia, Rizzoli, Milano.

GIAOUTZI M., NIJKAMP P. (1993), Decision support model for sustainable development, Aldershot, Avebury

GODFREY K., CLARKE J. (2000), The Tourism Development Handbook, Cassell, London.

GOLINELLI C.M., SIMONI M. (2006), "Gli strumenti del marketing turistico e territoriale", Sinergie. Rapporti di ricerca, vol. 2, n. 23, pp. 61-78.

GOLINELLI G.M. (2004), Ridefinire il valore della marca, Sinergie, n. 63, pp. 221-227.

GOLINELLI C., TRUNFIO M., LIGUORI M. (2006), "Governo e marketing del territorio", Sinergie, Rapporti di ricerca, vol. 2, n. 23, pp. 17-43.

GOODWIN H., FRANCIS, J. (2003), "Ethical and responsible tourism: consumer trend in the Uk", Journal of Vacation Marketing, vol. 9, n. 3, pp. 271-284.

HAUGLAND S.A., NESS H., GRONSETH B., AARSTAD J. (2011), "Development of tourism destinations. An Integrated Multilevel Perspective", Annals of Tourism Research, vol. 38, n. 1, pp. 268-290.

HUNTER C., GREEN H. (1995), Tourism and the environment: a sustainable relationship?, Routledge Press, London.

KAHNEMAN D., KRUEGER A.B. (2006), "Developments in the Measurement of Subjective Well-Being", Journal of Economic Perspectives, vol. 20, n.1, pp. 3-24.

KASPAR C. (1995), Management im Tourismus, Verlag Paul Haupt, Bern.

KAVARATZIS M. (2005), "Palce Branding", The Marketing Review, vol. 5, n. 4, pp. 329342.

LANCERINI E. (2005), “Territori lenti: contributi per una nuova geografia dei paesaggi abitati italiani", Territorio, n.34, pp. 9-15.

LAWS E. (1995), Tourist Destination Management, Issues Analysis and Policies, Routledge, London.

LAYARD R. (2006), Happiness. Lessons from a new science, Penguin, London.

LEIPER N. (1990), "Tourist Attraction System”, Annals of Tourism Research, vol. 17, n. 3, pp. 367-384.

MANENTE M., CERATO M. (2000), "Destination management per creare valore", in Pechlaner H., Weiermair K., Destination Management. Fondamenti di marketing e gestione delle destinazioni turistiche, Touring Editore, Milano.

MANZI E. (2001), "Paesaggio, turismo e sostenibilità ambientale. Qualche indicazione per una utopia necessaria”, Bollettino della Società Geografica Italiana, serie XII, vol. VI, pp. 389.403.

MARTINI U. (2005), Management dei sistemi territoriali. Gestione e marketing delle destinazioni turistiche, G. Giappicchelli, Torino.

MCKERCHER B. (1993), “Some fundamental truths about tourism: understanding tourism's social and environmental impacts", Journal of Sustainable Tourism, vol. 1, n. 1, pp. 616 . 
MIDDLETON V., HAWKINS R. (1998), Sustainable Tourism: A Marketing Perspective, Butterworth-Heinemann, Oxford.

MIHALIC T. (2000), "Environmental management of a tourist destination A factor of tourism competitiveness", Tourism Management, vol. 21, n. 1, pp. 65-78.

MURPHY P.E. (1985), Tourism: A Community Approach, Methuen, New York.

MUTTI A. (1998), Capitale sociale e sviluppo. La fiducia come risorsa, Il Mulino, Bologna.

PEARCE D.W., BARBIER E., MARKANDYA A. (1988), Sustainable Development and Cost Benefit Analysis, Paper 88/03. IIED/UCL London. Environmental Economics.

PECHLANER H., FISCHER E., HAMMAN E.M. (2005), "Creating the valuable basis of competitive advantages of destinations", in Keller P., Bieger T., Innovation in Tourism, AIEST, St. Gallen.

PETERS M., WEIERMAIR K., KATAWANDEE P. (2006), "Strategic Brand Management of tourism destination", in Keller P., Bieger T., Marketing Efficieny in Tourism, Schmidt -AIEST, Berlin.

PHILLIPS P.A., MOUTINHO L. (1998), Strategic planning systems in hospitality and tourism, CABI publishing, Wallingford, Oxon

PICHIERRI A. (1995), "Stato e identità economiche regionali", Stato e Mercato, n. 44, pp. 213-229.

PFOOR C. (2002), "The Markets and the Shakers of Tourism Policy in the Northern Territory of Australia: a Policy Network of Actors and Their Relationship Constellations", Journal of Hospitality and Tourism Management, vol. 9, n. 2, pp. 134-151.

POON A. (1993), Tourism, technology and competitive strategies, CABI Publishing, Wallingford, Oxon.

PUTNAM R. (1993), La tradizione civica nelle regioni italiane, Mondadori, Milano.

PRISKIN J. (2001), "Assessment of natural resources for nature-based tourism: the case of the Central Coast Region of Western Australia", Tourism Management, vol. 22, n. 6, pp. 637-648.

RITCHIE J.R.B., CROUCH G.I. (2000), "The competitive destination: a sustainable perspective", Tourism Management, vol. 21, n. 1, pp. 1-7.

ROBSON J., ROBSON I. (1996), "From Stakeholders to Stakeholders", Tourism Management, vol. 17, n. 7, pp. 533-540.

SAUTTER E.T., LEISEN B. (1999), "Managing Stakeholders", Annals of Tourism Research, vol. 26, n. 2, pp. 321-328.

SAMBRI C., PEGAN G. (2008), "La domanda di turismo responsabile. Un'indagine esplorativa sui turisti italiani nel nord-est", Mercati e competitività, n. 1, pp. 65-92.

SCOTT N., BAGGIO R., COOPER C. (2008), Network analysis and tourism, Channel View publication, Clevedon.

SEN A. (1999), Development as Freedom, Oxford University Press, Oxford.

SHMELEV S., RODRIGUEZ-LABAJOS B. (2009), "Dynamic multidimensional assessment of sustainability at the macro level: The case of Austria", Ecological Economics, vol. 68 , n. 10 , pp. $2560-2573$

SIMMONS D.G. (1994), "Community Participation in Tourism Planning", Tourism Management, vol. 15, n. 2, pp. 98-108.

STIGLITZ J., SEN A., FITOUSSI J. (2009), Report by the Commission on the Measurement of Economic Performance and Social Progress.

TIMOTHY D.J. (1999), Participatory Planning: a view of tourism in Indonesia, Annals of Tourism Research, vol. 26, n. 2, pp. 371-391.

TRIGILIA C. (1999), “Capitale sociale e sviluppo locale”, Stato e mercato, n. 57, pp. 419 440. 
VELLAS F., BÈCHEREL L. (1999), The international marketing of travel and tourism. A strategic approach, Macmillan Press, London.

WEAVER D.B., LAWTON L.J. (2007), "Twenty years on: the state of contemporary ecotourism research", Tourism Management, vol. 28, n. 5, pp. 1168-1179.

WTO (2004), Guida degli indicatori di sviluppo sostenibile per le destinazioni turistiche, traduzione italiana a cura della Provincia di Rimini. 
